\title{
One giant leap for Cas-kind
}

the MIMIVIRE
system is
suggested to
represent a
novel form
of adaptive
immunity

First reported in 2003, 'giant' viruses are double-stranded DNA viruses that are notable for the presence of several biological features that are usually found in cells rather than viruses, such as an extremely large genome and vulnerability to parasitism by phages ('virophages'). Levasseur et al. now describe MIMIVIRE, an antivirophage defence system that, through its seeming homology to bacterial and archaeal CRISPR-Cas systems, provides an intriguing new example of the unusual biology of giant viruses.

Giant viruses and virophages are produced in virion factories, which are specialized compartments of infected amoebae. The observation that virophages impair the reproduction of giant viruses raises the possibility that antivirophage defence systems may exist, analagous to the

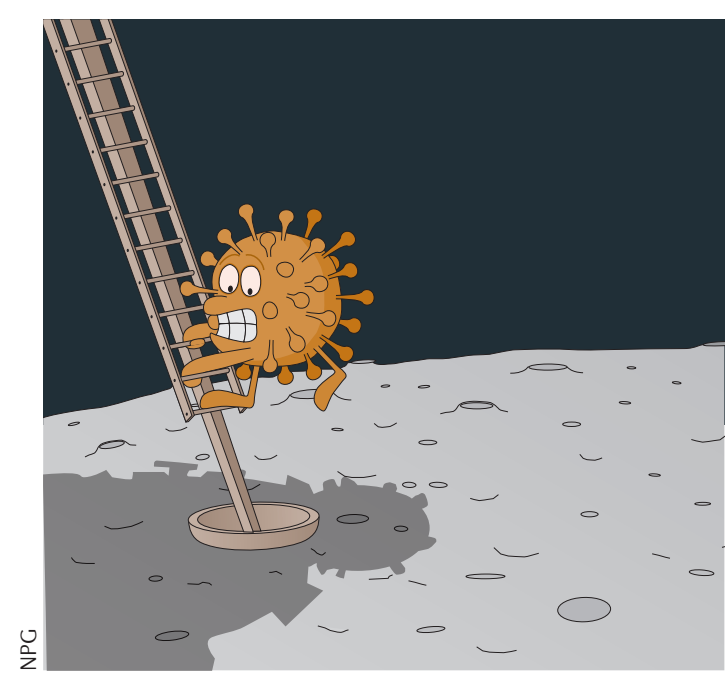

innate or adaptive immune systems used by bacteria and archaea to counter invading phages. Indeed, the Zamilon virophage, which is a parasite of the mimivirus family of giant viruses, infects virion factories that produce the $\mathrm{B}$ lineage and $\mathrm{C}$ lineage of mimivirus but not the A lineage, which suggests that A lineage mimiviruses may have acquired immunity to Zamilon.

Levasseur et al. hypothesized that the immunity to Zamilon might be reminiscent of CRISPR-Cas immunity, in which Cas proteins degrade specific nucleic acid sequences that have been committed to genomic memory in a CRISPR array. Accordingly, the genomes of 59 mimivirus strains, including 28 from the A lineage, 8 from the $\mathrm{B}$ lineage and 23 from the $\mathrm{C}$ lineage, were searched for traces of the Zamilon genome. Four Zamilon insertions (one $28 \mathrm{bp}$ sequence and three truncated, $15 \mathrm{bp}$ repeats of this sequence) were present in all of the genomes from the immune A lineage, but none of the genomes from the susceptible $\mathrm{B}$ lineage and $\mathrm{C}$ lineage. Importantly, knockdown of the ORF containing the four repeats by siRNA was sufficient to render A lineage mimiviruses susceptible to Zamilon, which confirmed the role of this locus in immunity to the virophage. Therefore, the four Zamilon repeats may represent an immune memory stored by an uncharacterized mimivirus adaptive immune system, which the authors termed 'MIMIVIRE'.
To identify putative components of the MIMIVIRE system, ORFs in the vicinity of the Zamilon insertions were queried for homology to genes of known function. Four ORFs were predicted to encode proteins with homology to Cas proteins, including two with homology to Cas3, a helicase; one with homology to Cas4, an exonuclease; and one with homology to RNase III, which processes transcribed CRISPR loci. All four of these ORFs are conserved in mimiviruses. Knockdown by siRNA confirmed that two of the ORFs, one with homology to Cas3 and one with homology to Cas4, were essential for the immunity of A lineage mimiviruses to Zamilon. Furthermore, functional assays using exogenous expression in Escherichia coli confirmed that the two ORFs encode enzymes with helicase and exonuclease activities, which the authors propose to be components of the MIMIVIRE system.

Owing to the strategy of storing a sequence memory in the MIMIVIRE locus and the homology of MIMIVIRE components to Cas3 and Cas4, the MIMIVIRE system is suggested to represent a novel form of adaptive immunity that, although present in viruses, may resemble the CRISPR-Cas systems found in bacteria and archaea.

Naomi Attar

ORIGINAL ARTICLE Levasseur, A. et al. MIMIVIRE is a defence system in mimivirus that confers resistance to virophage. Nature http://dx.doi. org/10.1038/nature17146 (2016) 